\title{
Multi-criteria decision making in supply chain management based on inventory levels, environmental impact and costs
}

\author{
Žic, J. ${ }^{\mathrm{a},{ }^{*}}, \check{Z ̌ i c}_{\mathrm{ic}} \mathbf{S} .^{\mathrm{a}}$ \\ aUniversity of Rijeka, Faculty of Engineering, Rijeka, Croatia
}

\begin{abstract}
A B S T R A C T
Supply chains in a global business environment operate within conflicting aspects. This research analyses correlation and interdependencies between inventory levels, costs and greenhouse gas emissions from replenishments within supply chain echelon. A simulation-based inventory optimisation conducted on 4000 experiments assumes the conditions of stochastic market demand, $(R, s, S)$ inventory policy, target fill rates, predefined lead times and closing days constraint. It verifies the influence of operational and logistic decisions such as frequency of inventory replenishments or vehicle size selection on management objectives. Besides determining the best individual results for the objectives of minimum inventory levels, total costs and emissions, the overall best solutions in terms of three decision models - uniformly valued, cost-oriented and environmentally responsible model, were determined using multi-criteria decision-making methodology. These models are relevant for both scientific and practical managerial settings due to the evident lack of research simultaneously analysing inventory, cost and environmental performances of $(R, s, S)$ policy. This study confirms that it is crucial in practice to perform an extensive simulation experiment analysis for each product to be able to determine its optimal settings. Inventory management software should have a direct influence on operational decisions in order to reduce costs or emissions within the same fill rate.
\end{abstract}

ARTICLE INFO

Keywords:

Green supply chain;

Multi-criteria decision making;

Environmental impact;

Costs;

Inventory levels

*Corresponding author: jzic@riteh.hr

(Žic, J.)

Article history:

Received 17 March 2020

Revised 17 July 2020

Accepted 20 July 2020

\section{References}

[1] Cetinkaya, B., Cuthbertson, R., Ewer, G., Klaas-Wissing, T., Piotrowicz, W., Tyssen, C. (2011). Sustainable supply chain management, Springer-Verlag, Berlin Heidelberg, Germany, doi: 10.1007/978-3-642-12023-7.

[2] Shapiro, J. (2001). Modelling the supply chain, Duxbury Press, Pacific Grove, California, USA.

[3] Ferretti, I., Zanoni, S., Zavanella, L., Diana, A. (2007). Greening the aluminium supply chain, International Journal of Production Economics, Vol. 108, No. 1-2, 236-245, doi: 10.1016/j.ijpe.2006.12.037.

[4] Srivastava, S.K. (2007). Green supply chain management: a state-of-the-art literature review, International Journal of Management Reviews, Vol. 9, No. 1, 53-80, doi: 10.1111/i.1468-2370.2007.00202.x.

[5] Christoper, M., Towill, D.R. (2000). Supply chain migration from lean and functional to agile and customised, Supply Chain Management, Vol. 5, No. 4, 206-213, doi: 10.1108/13598540010347334.

[6] Bonney, M., Jaber, M.Y. (2011). Environmentally responsible inventory models: non-classical models for a nonclassical era, International Journal of Production Economics, Vol. 133, No. 1, 43-53, doi: 10.1016/i.ipe.2009.10. $\underline{033}$.

[7] Wahab, M.I.M., Mamun, S.M.H., Ongkunaruk, P. (2011). EOQ models for a coordinated two-level, Journal of Production Economics, Vol. 134, No. 1, 151-158, doi: 10.1016/i.ijpe.2011.06.008.

[8] Demir, E., Bektaş, T., Laporte, G. (2014). A review of recent research on green road freight transportation, European Journal of Operational Research, Vol. 237, No. 3, 775-793, doi: 10.1016/i.ejor.2013.12.033.

[9] Venkat, K., Wakeland, W. (2006). Is lean necessarily green?, In: Proceedings of the 50th Annual Meeting of the International Society for the Systems Sciences, Sonoma, California, USA. 
[10] Oršič, J., Rosi, B., Jereb, B. (2019). Measuring sustainable performance among logistic service providers in supply chains, Tehnički Vjestnik - Technical Gazette, Vol. 26, No. 5, 1478-1485, doi: 10.17559/TV-20180607112607.

[11] Somboonwiwat, T., Khompatraporn, C., Miengarrom, T., Lerdluechachai, K. (2018). A bi-objective environmentaleconomic optimisation of hot-rolled steel coils supply chain: A case study in Thailand, Advances in Production Engineering \& Management, Vol. 13, No. 1, 93-106, doi: 10.14743/apem2018.1.276.

[12] Balamurugan, T., Karunamoorthy, L., Arunkumar, N., \& Santhosh, D. (2018). Optimisation of inventory routing problem to minimise carbon dioxide emission, International Journal of Simulation Modelling, Vol. 17, No. 1, 4254, doi: 10.2507/IISIMM17(1)410.

[13] Benjaafar, S., Li, Y., Daskin, M. (2013). Carbon footprint and the management of supply chains: Insights from simple models, IEEE Transactions on Automation Science and Engineering, Vol. 10, No. 1, 99-116, doi: 10.1109/ TASE.2012.2203304.

[14] Kiesmüller, G.P., de Kok, A.G., Dabia, S. (2011). Single item inventory control under periodic review and a minimum order quantity, International Journal of Production Economics, Vol. 133, No. 1, 280-285, doi: 10.1016/i.ijpe. 2010.03.019.

[15] Sani, B., Kingsman, B.G. (1997). Selecting the best periodic inventory control and demand forecasting methods for low demand items, Journal of the Operational Research Society, Vol. 48, No. 7, 700-713, doi: 10.1057/ palgrave. jors. 2600418.

[16] Jian, M., Wang, Y.L. (2018). Decision-making strategies in supply chain management with a waste-averse and stockout-averse manufacturer, Advances in Production Engineering \& Management, Vol. 13, No. 3, 345-357, doi: 10.14743/apem2018.3.295.

[17] Kapalka, B.A., Katircioglu, K., Puterman, M.L. (1999). Retail inventory control with lost sales, service constraints, and fractional lead times, Production and Operations Management, Vol. 8, No. 4, 393-408, doi: 10.1111/i.19375956. 1999.tb00315.x.

[18] Bijvank, M., Vis, I.F.A. (2012). Lost-sales inventory systems with a service level criterion, European Journal of Operational Research, Vol. 220, No. 3, 610-618, doi: 10.1016/i.ejor.2012.02.013.

[19] Bijvank, M. (2014). Periodic review inventory systems with a service level criterion, Journal of the Operational Research Society, Vol. 65, No. 12, 1853-1863, doi: 10.1057/iors.2013.160.

[20] Gocken, M., Dosdogru, A.T., Boru, A. (2017). Optimization via simulation for inventory control policies and supplier selection, International Journal of Simulation Modelling, Vol. 16, No. 2, 241-252, doi: 10.2507/IISIMM16 (2) 5.375 .

[21] Tang, S., Wang, W., Yan, H., Hao, G. (2015). Low carbon logistics: Reducing shipment frequency to cut carbon emissions, International Journal of Production Economics, Vol. 164, 339-350, doi: 10.1016/i.ipe.2014.12.008.

[22] Akhtari, S., Sowlati, T., Siller-Benitez, D.G., Roeser, D. (2019). Impact of inventory management on demand fulfilment, cost and emission of forest-based biomass supply chains using simulation modelling, Biosystems Engineering, Vol. 178, 184-199, doi: 10.1016/i.biosystemseng.2018.11.015.

[23] Digiesi, S., Mossa, G., Mummolo, G. (2013). A sustainable order quantity model under uncertain product demand, IFAC Proceedings Volumes, Vol. 46, No. 9, 664-669, doi: 10.3182/20130619-3-RU-3018.00444.

[24] Battini, D., Persona, A., Sgarbossa, F. (2014). A sustainable EOQ model: Theoretical formulation and applications, International Journal of Production Economics, Vol. 149, 145-153, doi: 10.1016/i.ijpe.2013.06.026.

[25] Chen, X., Benjaafar, S., Elomri, A. (2013). The carbon-constrained EOQ, Operations Research Letters, Vol. 41, No.2, 172-179, doi: 10.1016/i.orl.2012.12.003.

[26] Konur, D., Schaefer, B. (2014). Integrated inventory control and transportation decisions under carbon emissions regulations: LTL vs. TL carriers, Transportation Research, Vol. 68, 14-38, doi: 10.1016/j.tre.2014.04.012.

[27] Darvish, M., Archetti, C., Coelho, L.C. (2019). Trade-offs between environmental and economic performance in production and inventory-routing problems, International Journal of Production Economics, Vol. 217, 269-280, doi: 10.1016/j.ijpe.2018.08.020.

[28] Yu, W., Hou, G., Xia, P., Li, J. (2019). Supply chain joint inventory management and cost optimisation based on ant colony algorithm and fuzzy model, Tehnički Vjestnik - Technical Gazette, Vol. 26, No. 6, 1729-1737, doi: 10.17559/TV-20190805123158.

[29] Janssen, L., Sauer, J., Claus, T., Nehls, U. (2018). Development and simulation analysis of a new perishable inventory model with a closing days constraint under non-stationary stochastic demand, Computers \& Industrial Engineering, Vol. 118, 9-22, doi: 10.1016/i.cie.2018.02.016.

[30] Bozorgi, A., Pazour, J., Nazzal, D. (2014). A new inventory model for cold items that considers costs and emissions, International Journal of Production Economics, Vol. 155, 114-125, doi: 10.1016/j.ijpe.2014.01.006.

[31] Eurostat. Greenhouse gas emission statistics - Emission inventories, from https://ec.europa.eu/eurostat/ statistics-explained/pdfscache/1180.pdf, accessed February 11, 2020.

[32] European Environmental Agency (EEA). Greenhouse gas emissions from transport in Europe, from https:// www.eea.europa.eu/data-and-maps/indicators/transport-emissions-of-greenhouse-gases/transport-emissions-ofgreenhouse-gases-12, accessed February 11, 2020.

[33] European Committee for Standardization (CEN), (2012). Methodology for calculation and declaration of energy consumption and GHG emissions of transport services (freight and passengers), SS-EN 16258:2012, CEN, Brussels, Belgium, from:https://www.sis.se/en/produkter/sociology-services-company-organization/services/servicesfor-consumers/ssen162582012/, accessed February 10, 2020.

[34] ifeu, INFRAS, IVE. Ecotransit world: Methodology and data - Update 2019, from https://www.ecotransit.org/download/EcoTransIT_World_Methodology_Data_Update_2019.pdf, accessed February 10, 2020. 
[35] Urban, T.L. (1998). An inventory - Theoretic approach to product assortment and shelf-space allocation, Journal of Retailing, Vol. 74, No. 1, 15-35, doi: 10.1016/S0022-4359(99)80086-4. 


\section{APEM}

\section{Večkriterijsko odločanje pri upravljanju dobavne verige na podlagi ravni zalog, vpliva na okolje in stroškov}

Žic, J. ${ }^{\mathrm{a},{ }^{*}}$, Žic, $\mathbf{S} .^{\mathrm{a}}$

${ }^{a}$ University of Rijeka, Faculty of Engineering, Rijeka, Croatia

\section{POVZETEK}

Dobavne verige $\mathrm{v}$ globalnem poslovnem okolju delujejo $\mathrm{v}$ nasprotujočih si vidikih. Ta raziskava analizira korelacije in soodvisnosti med ravnmi zalog, stroški in emisijami toplogrednih plinov, zaradi dopolnitev v delih dobavne verige. Simulacija optimizacije zalog, izvedena na 4000 eksperimentih, predpostavlja pogoje stohastičnega povpraševanja na $\operatorname{trgu},(R, S, S)$ politiko zalog, ciljne stopnje polnjenja, vnaprej določene roke in omejitve zaključnih dni. Preveri se vpliv operativnih in logističnih odločitev, kot sta pogostost dopolnjevanja zalog ali izbira velikosti vozila na cilje upravljanja. Poleg določanja najboljših posameznih rezultatov za zagotovitev minimalnih zalog, skupnih stroškov in emisij so bile $\mathrm{z}$ uporabo večkriterijske metodologije odločanja določene tudi skupne najboljše rešitve $v$ smislu treh modelov odločanja: enotnega vrednotenja, stroškovno naravnanega in okoljsko odgovornega modela. Ti modeli so pomembni iz vidika znanstvene in praktične vodstvene orientacije, zaradi očitnega pomanjkanja raziskav, ki hkrati analizirajo zaloge, stroške in okoljske učinke politike $(R, s, S)$. Ta študija potrjuje, da je v praksi ključnega pomena izvesti obsežno analizo simulacijskega eksperimenta za vsak izdelek, da lahko določimo njegove optimalne nastavitve. Programska oprema za upravljanje zalog mora imeti neposreden vpliv na operativne odločitve, da se znižajo stroški ali emisije znotraj iste stopnje polnjenja.

\section{PODATKI O ČLANKU}

Ključne besede:

Zelena dobavna veriga;

Večkriterijsko odločanje;

Vpliv na okolje;

Stroški;

Raven zalog

*Kontaktna oseba: jzic@riteh.hr

(Žic, J.)

Zgodovina članka:

Prejet 17. marca 2020

Popravljen 17. julija 2020

Sprejet 20. julija 2020 